\title{
On the Real-time Airport Parking Position Reassignment Problem
}

\author{
Jun $\mathrm{Bi}^{1, \mathrm{a}}$, Qianwen $\mathrm{Li}^{1, \mathrm{~b},{ }^{*}}$ and Xiaomei Zhao ${ }^{1, \mathrm{c}}$ \\ ${ }^{1}$ School of Traffic and Transportation, Beijing Jiaotong University, Beijing 100044, China \\ abilinghc@163.com, b15120758@bjtu.edu.cn, ’xmzhao@bjtu.edu.cn
}

Keywords: Parking position reassignment; Real-time; Airport operations; Optimization

\begin{abstract}
In actual day to day airport operations, interference (such as the flight ahead of schedule, delay and cancellation) is a common occurrence. This article is mainly focus on how to quickly and reasonably solve the real-time parking position reassignment problem caused by interference. A real-time airport parking position assignment model solved by GUROBI optimization software is built and the objective of this model is to minimize the number of passengers assigned to apron and the number of inconsistency between the reassignment scheme and the original one. Finally, the result of a test case verifies that the proposed approach performs well.
\end{abstract}

\section{Introduction}

Interference (such as the flight ahead of schedule, delay and cancellation) is a frequent occurrence in airport daily operations, which forced the airport authority to reassign aircraft to different parking positions in real-time. Since these aircraft information may vary and is updated frequently, the reassignment process must be immediately and reasonably. And traditional methods are not appropriate for solving real-time parking position reassignment problem owing to time-consuming.

Beyond that, with the airport flight traffic is growing, fixed and limited resources such as the airport runways, taxiways and parking positions are falling short of needs while activity planning is increasingly crucial and complex. Fig. 1 illustrates the two types of possible parking positions: airbridge parking position and remote parking position (apron). Airports and airlines usually prefer airbridge parking positions because they connect to an airport terminal passenger gate through airbridge and are more convenient for passengers. In our partner airport, the number of passengers allocated to apron has increased in the last years, which increases the bus transfer costs and leads to the deterioration in service quality. Consequently, under the premise of ensuring the safety of flight and landing, through the rational allocation of parking positions to improve the efficiency of the airport and the level of service to passengers, which has become the airport operators need to further study and solve the problem.

In this paper we deal with the Real-Time Parking Positions Reassignment Problem which is similar to the Airport Gate Assignment Problem (AGAP). The Parking Positions Allocation Problem refers to seeks feasible aircraft-to-parking position assignments with satisfying some constraints to minimize the number of passengers assigned to apron and the number of inconsistency between the reassignment scheme and the original one.

AGAP has already been studied by many researchers. In order to minimize the variance of idle times as the optimization objective, the corresponding models are built on the basis of branch and bound method, genetic algorithm and other methods to achieve the robustness goal of the gate assignment by Bolat A[1-2]. J Xu and G Bailey (2001) consider an airport gate assignment problem that dynamically assigns airport gates to scheduled flights based on passengers' daily origin and destination flow data. They reformulate it as a mixed 0-1 integer problem with a linear objective function and constraints. And they design a simple tabu search meta-heuristic to solve the problem [3]. Ding et al. (2004) analyses the shortcomings in the solve approach given by $\mathrm{Xu}$ and Bailey and then put forward a new heuristics for over-constrained flight to gate assignments [4]. S Yan and $\mathrm{CH}$ Tang (2007) thought stochastic flight delays that occur in actual operations also have to be taken into account. They develop a heuristic approach embedded in a framework designed to help the airport authorities make airport gate assignments that are sensitive to stochastic flight delays [5]. $\mathrm{CH}$. Tang designed a gate reassignment model to deal with temporary gate shortages and stochastic 
flight delays [6]. H Zhao and L Cheng proposed a mixed integer model and used colony algorithm to solve airport gate assignment problem [7].

Many scholars have made a great deal of attention to AGAP and basic models for real-time allocation of parking positions or parking position pre-assignment are also proposed. However, how to quickly and reasonably solve the real-time parking position reassignment problem caused by interference and minimize the number of inconsistencies between the reassignment scheme and the original one are rarely involved.

This article is mainly focus on how to quickly and reasonably solve the real-time parking position reassignment problem caused by interference. And this paper has been organized in several sections. In the next section, we give a formulation of the Parking Positions Allocation Problem as we model it. In Section 3, we design a two-phase computerized method to solve real-time parking position assignment. In Section 4, computational results and comparisons are presented and in Section 5, the conclusion of this paper is given.

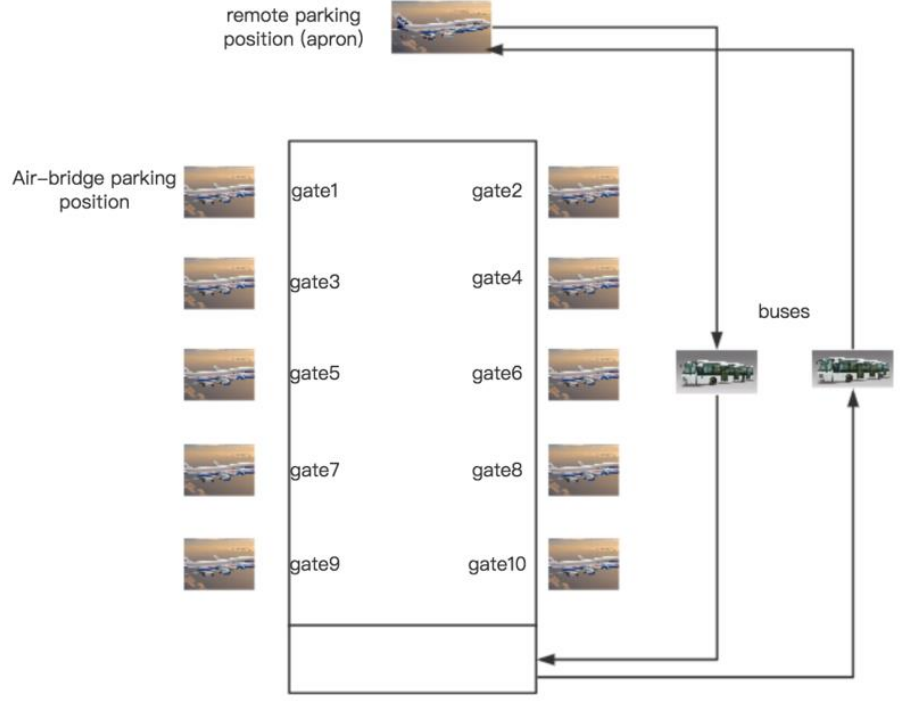

Figure 1. Airport parking positions

\section{Model Development}

In this section, we formulated the problem as an integer programming model with some constrains and the model is devoted to minimization of the passenger assigned to apron. Some constraints are necessary to this problem and mainly include two points: each aircraft must be assigned to only one parking position and no two aircraft with overlapping parking position occupation times are assigned to the same parking position at the same time.

Besides the above-mentioned constrains, we also need to take into account some additional restrictions related to the airport facilities. First, Aircraft-parking position compatibility must be considered. For example, due to the space restrictions related to the sizes of the available parking positions and aircrafts, some parking positions are forbidden to some aircrafts of specific types. Next, the process of an aircraft parking contains three parts: disembarkation of the arrival flight, waiting, and embarkation of the departure flight. During the waiting period, airport operators may decide to tow (move) aircraft from apron to air-bridge parking position for improving the airbridge utilization. However, note that only if the duration of the waiting period is long enough then we can move the aircraft through the towing tractor. Finally, adjacency conflicts must be taken into account. For example, two large aircraft can't be assigned to adjacent parking positions concurrently.

Mathematical Notation. The notations used are listed as followed. Sets:

F: set of aircraft;

M: set of aircraft which was assigned to on previous run and moves are allowed for the aircraft; 
$\mathbf{N}$ : set of aircraft which have already arrived and moves are forbidden for the aircraft;

R: set of air-bridge parking position;

A: set of remote parking position;

G: AUR

Parameters:

$t_{i}^{a}$ : The estimated time of arrival of aircraft $i, i \in \mathrm{F}$;

$t_{i}^{d}$ : The estimated time of departure of aircraft $i, i \in \mathrm{F}$;

$T_{\text {buffer }}$ : The buffer time between two aircraft assigned to the same parking position;

$N_{i}^{p}$ : The number of passengers of aircraft $i, i \in \mathrm{F}$;

$x_{i k}^{\prime}$ : Binary parameter equal to 1 if aircraft $\mathrm{i}$ was assigned to original parking position $k$ on previous run, $i \in \mathrm{M}, k \in \mathrm{G}$;

$B_{k}$ : Binary parameter equal to 1 if parking position $k$ belong to $\mathrm{A}, k \in \mathrm{G}$;

$H_{k}^{s}$ : The idle start time of parking position $k, k \in \mathrm{G}$;

$A_{k l}$ : Binary parameter equal to 1 if parking position $k$ adjacent to parking position $l, k, l \in \mathrm{G}$;

$Q_{i k}$ : Binary parameter equal to 1 if aircraft $i$ is compatible with parking position $k, k \in \mathrm{G}, \mathrm{i} \in \mathrm{F}$;

$P_{i j}$ : Binary parameter equal to 1 if the type of aircraft $i$ conflicts with the type of aircraft $j$ while aircraft $i, j$ assigned to adjacency parking positions and aircraft $j$ belongs to $\mathrm{R}_{\mathrm{i}}, i, j \in \mathrm{F}$;

$R_{i}$ : Set of aircraft conflicted with aircraft $i$;

Variables:

$x_{i k}$ : Binary parameter equal to 1 if aircraft $\mathrm{i}$ is assigned to parking position $\mathrm{k}, \mathrm{i} \in \mathrm{M}, \mathrm{k} \in \mathrm{G}$;

Mathematical Model. Objective of this model is to minimize the number of passengers assigned to apron and minimize the number of inconsistency value which indicates an aircraft is reassigned to a parking position different than the original one. The weights are $\alpha, \beta$. And the mathematical model for Parking Positions Allocation Problem is now presented as follows:

$\operatorname{minf} \alpha \sum_{i=1}^{m} \sum_{k=1}^{n} x_{i k} \times B_{k} \times N_{i}^{p}+\beta \sum_{i=1}^{m} \sum_{k=1}^{n}\left(x_{i k}-x_{i k}^{\prime}\right)^{2}$

Subject to:

$$
\begin{array}{ll}
\sum_{k=1}^{n} x_{i k}=1 \quad \forall i \in \mathbf{F}, \forall k \in \mathbf{G} \\
\sum_{j \in R(i)} x_{j k}+x_{i k} \leq 1 \quad \forall i \in \mathbf{F}, \forall k \in \mathbf{G} \\
x_{i k} P_{i j}+x_{j l} P_{i j} \leq 1 \quad \forall A_{k l}=1, \forall i, j \in \mathbf{F} \text { and } i \neq j, \forall k, l \in \mathbf{G} \text { and } k \neq l \\
x_{i k}=0 & \forall Q_{i k}=0, \forall i \in \mathbf{F}, \forall k \in \mathbf{G} \\
H_{k}^{s} \leq t_{i}^{a} & \forall i \in \mathbf{F}, \forall k \in \mathbf{G} \\
x_{i k}=0 & \forall k \in\left\{k \mid x_{i k}^{\prime}=0 \text { and } B_{k}=0\right\}, \forall i \in \mathbf{M} \\
x_{i k}=1 & \forall x_{i k}^{\prime}=1, \forall i \in \mathbf{N}, \forall k \in \mathbf{G}
\end{array}
$$

Constraint (2) ensures that each aircraft must be allocated to only one certain parking position. Constraint (3) specifies that two aircraft's schedule can't overlap if they are arranged to the same parking positions. Constraint (4) restricts that two large aircraft can't be assigned to adjacent parking positions concurrently. Constraint (5) ensures that aircraft-parking position compatibility must be satisfied. Constraint (6) guarantees that each aircraft must be allocated to the parking position whose idle start time doesn't exceed the estimated time of departure of the aircraft. Constraint (7) ensures that only can move the aircraft from apron to air-bridge parking position through the towing tractor. Constraint (8) ensures that those arrived aircraft for which moves are forbidden can only assignment to their original parking position. 


\section{Solution Strategy}

For computational purposes, we develop a two-phase computerized method which includes the preassignment and reassignment phases to solve real-time parking position assignment.

Phase 1: Pre-assignment. Consider the following.

The first phase's execution time is the start time of the daily airport operations. The relevant elements, including aircraft information, the available parking positions and parameters are provided in the preprocessing phase. Then, obtain an optimized pre-assignment result based on relevant elements.

Phase2:Reassignment.Consider the following.

This phase is designed to automatically and quickly provide the reassignment result after preprocessing of the updated aircraft and parking position data until the end of daily operations, or until the last aircraft's parking position is fixed in daily operations. Time interval for updating data is set to be $30 \mathrm{~min}$.

Step1.Calculate the idle start time for each parking position according to the aircraft's arrival/departure time (The aircraft's parking position is fixed).

Step2.On the phrase of data preprocessing, we need to update the aircraft information such as whether it can be moved, whether it has arrived, arrival time, and departure time.

Step3.Obtain a new optimized reassignment result based on updated elements, using the optimization software GUROBI.

\section{Computational Study}

In order to solve the model as mentioned in the last sect effectively, the optimization software GUROBI is taken into application. And we performed computational study based on our partner airport.

The planned parking position assignment data used for computational study were based on the operating day, December 24, 2016 and included 81 aircraft and 5 aircraft types. The Partial aircraft information is shown in Table 1. There were 19 parking positions in operation, of which 10 were remote parking positions.

Table 1 Part of aircraft information

\begin{tabular}{|c|c|c|c|c|c|c|}
\hline $\begin{array}{c}\text { Aircraft } \\
\text { ID }\end{array}$ & towed & Arrival time & Departure time & $\begin{array}{c}\text { Aircraft } \\
\text { type }\end{array}$ & Passenger & $\begin{array}{c}\text { Actual parking } \\
\text { position }\end{array}$ \\
\hline B1 & yes & $12 / 2322: 46$ & $12 / 247: 05$ & B737 & 232 & 10 \\
\hline D2 & no & $12 / 248: 29$ & $12 / 249: 45$ & A320 & 96 & 10 \\
\hline D75 & no & $12 / 2410: 32$ & $12 / 2411: 40$ & B737 & 284 & 9 \\
\hline D35 & no & $12 / 2411: 30$ & $12 / 2412: 15$ & A320 & 29 & 15 \\
\hline A60 & no & $12 / 2413: 34$ & $12 / 2418: 50$ & A320 & 102 & 6 \\
\hline
\end{tabular}

After data preprocessing phase, we used software to obtain an optimized pre-assignment result listed in Table 2. In pre-assignment results, aircraft A19, A27, A55, A57, B59 need to be towed from apron to air-bridge parking position by towing tractor. Compared with parking position allocation scheme in actual operations, the number of passengers in pre-assignment results with the model and program proposed in this paper is increased from 11591 to 14469 , a growth of $24.82 \%$. 
Table 2 Pre-assignment results

\begin{tabular}{|c|c|c|}
\hline $\begin{array}{c}\text { Parking position } \\
\text { ID }\end{array}$ & $\begin{array}{c}\text { Aircrafts assigned to the } \\
\text { parking position }\end{array}$ & $\begin{array}{c}\text { Passengers assigned to } \\
\text { air-bridge parking position }\end{array}$ \\
\hline 1 & & 1286 \\
\hline 2 & & 1757 \\
\hline 3 & A19, A36, D21, D25, D50, D66, D67 & 1677 \\
\hline 4 & & 2099 \\
\hline 5 & B58, D32, D46, A20, B77, D16, C17, D26, A80, A52 & 758 \\
\hline 6 & B59, B45, B61, B62, B64, B8, D71,D51 & 1790 \\
\hline 7 & A27, C68, A76, A54 & 1612 \\
\hline 8 & A57, D74, C12, D13, D47, D37, A15, D78, D65, D42 & \\
\hline 9 & B1, D2, A28, D29, D7, B79, D41, D10, B73 & \\
\hline 10 & A11, D3, D4, C5, A6, B70, A40, A81 & 1977 \\
\hline 11 & D24, B30 & 1513 \\
\hline 12 & A23 & \\
\hline 13 & & \\
\hline 14 & A53, B56 & 14469 \\
\hline 15 & D39 & \\
\hline 16 & A55, B44, D34, D35, D14, A48, D49, C9, B72 & \\
\hline 17 & A60 & \\
\hline 18 & & \\
\hline 19 & & \\
\hline Total & & \\
\hline
\end{tabular}

Due to weather, traffic control and other reasons, the following aircraft's arrival/departure time are altered compared with the original flight schedule as is shown in Table 3.

Table 3 Irregular aircrafts information

\begin{tabular}{cccc}
\hline $\begin{array}{c}\text { Aircraft } \\
\text { ID }\end{array}$ & state & $\begin{array}{c}\text { Arrival } \\
\text { delay }\end{array}$ & $\begin{array}{c}\text { Departure } \\
\text { delay }\end{array}$ \\
\hline B1 & Cancelled & & \\
D47 & Advanced & $-15 \mathrm{~min}$ & -- \\
C9 & Due & $+20 \mathrm{~min}$ & $+25 \mathrm{~min}$ \\
C12 & Due & $+40 \mathrm{~min}$ & $+45 \mathrm{~min}$ \\
D35 & Advanced & $-10 \mathrm{~min}$ & -- \\
D67 & Due & $+70 \mathrm{~min}$ & $+70 \mathrm{~min}$ \\
A20 & Due & $+35 \mathrm{~min}$ & $+35 \mathrm{~min}$ \\
B45 & Due & $+25 \mathrm{~min}$ & $+18 \mathrm{~min}$ \\
\hline
\end{tabular}

As described above, automatically and quickly provide the reassignment result after preprocessing of the updated irregular aircrafts information in Table 3 and parking position data. After running about 10 seconds, we can obtain the real-time reassignment result. And the total aircrafts reassigned parking position inconsistency between the reassignment scheme and the original one refers to Table 4. The reassignment results showed in Fig. 3. Due to the gap between the successive aircraft assigned to the same parking position is too small, some aircraft can't be seen clear in figure. As can be seen, when 8 of the 81 aircrafts in the model are disturbed, only 4 of the aircrafts have a real-time reassigned parking position which is different from the original parking position, and has less interference. 
Table 4 Parking positions inconsistencies

\begin{tabular}{|c|c|c|}
\hline Aircraft ID & $\begin{array}{c}\text { Original parking } \\
\text { position }\end{array}$ & $\begin{array}{c}\text { Reassignment of parking } \\
\text { position }\end{array}$ \\
\hline B73 & 10 & 9 \\
\hline C17 & 5 & 16 \\
\hline B72 & 16 & 11 \\
\hline C12 & 8 & 16 \\
\hline D34 & 16 & 12 \\
\hline
\end{tabular}

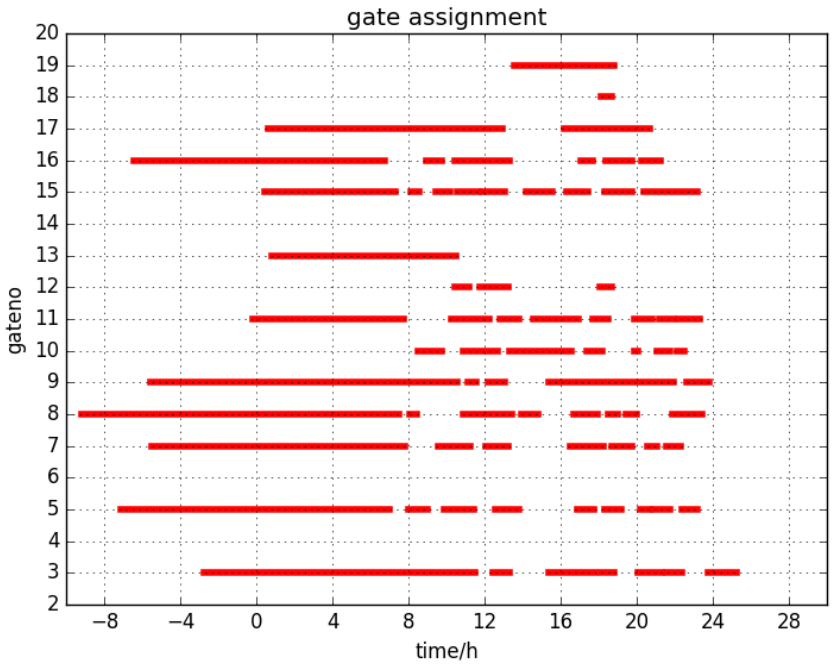

Figure 2. Figure of reassignment results

\section{Conclusions}

In this paper, we have shown a binary integer program model for the aircraft parking position reassignment problem that minimizes the number of passengers assigned to apron and the number of inconsistency between the reassignment scheme and the original one. For computational purposes, we develop a two-phase computerized method which includes the pre-assignment and reassignment phases to solve real-time parking position assignment. GUROBI and python were used to code the program which was applied to our partner airport and be illustrated to provide optimal solutions in a matter of seconds. In practice, this tool may be used by parking position managers to optimize the reassignment of aircraft to parking positions meet alterations in aircraft arrival/departure times in real-time.

\section{Acknowledgements}

This research is supported by Key research and development project of Shandong Province (2016GGX105004) and Kunming science technology project of Yunnan Province (2016X25).

\section{References}

[1] Bolat A: Assigning arriving flights at an airport to the available gates. Journal of the Operational Research Society, 1999, 50 (1) :23-34.

[2] Bolat A: Procedures for providing robust gate assignments for arriving aircrafts. European Journal of Operational Research, 2000, 120 (1):63-80.

[3] $\mathrm{J} \mathrm{Xu}$ and G Bailey, 2001. The airport gate assignment problem: mathematical model and a tabu search algorithm. In: 34th Hawaii International Conference on System Sciences (HICSS-34), vol. 3, Hawaii, 2001, pp. 3032 - 3040. 
[4] Ding H, Lim A, Rodrigues B and Zhu Y: New heuristics for over-constrained flight to gate assignments. Journal of the Operational Research Society, 2004, 55 (7) , pp. 760-768.

[5] S Yan and $\mathrm{CH}$ Tang: A heuristic approach for airport gate assignments for stochastic flight delays. European Journal of Operational Research, vol. 180, no. 2, pp. 547-567, 2007.

[6] CH. Tang: A gate reassignment model for the Taiwan Taoyuan airport under temporary gate shortages and stochastic flight delays. IEEE Transactions on Systems, Man, and Cybernetics A: Systems and Humans, vol. 41, no. 4, pp. 637-650.

[7] H Zhao and L Cheng: Ant colony algorithm and simulation for robust airport gate assignment. Mathematical Problems in Engineering, Volume 2014. 